\title{
COMPORTAMENTO DE TRAÇÃO DE COMPÓSITOS DE MATRIZ EPOXI REFORÇADOS COM TECIDO RAMIE *
}

\author{
Artur Camposo Pereira ${ }^{1}$ \\ Fabio Da Costa Garcia Filho ${ }^{2}$ \\ Noan Tonini Simonassi $i^{3}$ \\ Sérgio Neves Monteiro ${ }^{4}$
}

\section{Resumo}

As fibras naturais foram estudadas como um material de reforço alternativo para substituir o uso de fibras sintéticas. Com o objetivo de melhorar as propriedades dos compostos de fibras naturais, é investigado o uso de tecidos de fibras naturais, uma vez que proporcionam reforço multidirecional e permite a introdução de uma fração de volume de fibra maior. Neste trabalho, investigou-se o comportamento de tração de compósitos de matriz epóxi reforçados com diferentes frações de volume de tecido feito de rami, uma fibra lignocelulósica altamente resistente. Os espécimes foram feitos pressionando o tecido com resina epóxi para garantir a impregnação total, e depois cortando o tecido no formato do molde. Finalmente, as camadas de tecido foram colocadas no molde com a resina líquida e o catalisador. O material foi curado durante 24 horas e testado. Os resultados revelaram um aumento na resistência à tração e quase nenhum efeito no módulo de elasticidade com o aumento da fração do volume do tecido.

Palavras-chave: Fibras naturais, compósitos de rami e compósitos epóxi.

\section{TENSILE BEHAVIOR OF EPOXY MATRIX COMPOSITES REINFORCED WITH RAMIE FABRIC}

\section{Abstract}

Natural fibers have been studied as an alternative reinforcement material to replace the use of synthetic fibers. Aiming to improve the properties of natural fibers composites, it is investigated the use of natural fibers fabrics, since they provide multidirectional reinforcement and enables the introduction of a larger fiber volume fraction. In this work, it was investigated the tensile behavior of epoxy matrix composites reinforced with different volume fractions of fabric made of ramie, a highly resistant lignocellulosic fiber. The specimens were made by pressing the fabric with epoxy resin to guarantee total impregnation, and then cutting the fabric in the mold format. Finally, the fabric layers were placed into the mold with the liquid resin and catalyst. The material was cured for 24 hours and tested. The results revealed an increase in the tensile strength and almost no effect in the elastic modulus with the increase of fabric volume fraction.

Keywords: Natural fibers, ramie fabric composite, epoxy composites.

1 Engenheiro de Materiais, Doutorando em Ciência dos Materiais, IME - Instituto Militar de Engenharia, Praça General Tibúrcio, 80 22290-270, Rio de Janeiro, RJ, Brasil.

2 Engenheiro de Materiais, Mestrando em Ciência dos Materiais, IME - Instituto Militar de Engenharia, Praça General Tibúrcio, 80 22290-270, Rio de Janeiro, RJ, Brasil.

3 Engenheiro de Materiais, Doutorando em Ciência dos Materiais, IME - Instituto Militar de Engenharia, Praça General Tibúrcio, 80 22290-270, Rio de Janeiro, RJ, Brasil.

4 Engenheiro de Materiais, Seção de ensino de Ciência e engenharia de Materiais, IME - Instituto Militar de Engenharia, Praça General Tibúrcio, 80 22290-270, Rio de Janeiro, RJ, Brasil. 


\section{INTRODUÇÃO}

Com o rápido desenvolvimento da nossa sociedade moderna nas últimas décadas, muitos estudos foram realizados para desenvolver materiais que atendam as novas demandas. Neste contexto, é extremamente importante considerar não só os benefícios técnicos de projetos e pesquisas, mas também suas vantagens ambientais visando sua sustentabilidade a longo prazo. Uma das preocupações mais importantes é encontrar alternativas aos materiais não renováveis, que contribuem para a poluição do ar, para a escassez de recursos e para aumentar a quantidade de resíduos não biodegradáveis. Nesse cenário, as fibras naturais emergem como uma alternativa vantajosa [1-3].

Entre as fibras naturais, as lignocelulósicas devem ser destacadas por ter alta resistência mecânica. A presença de lignina e celulose torna as fibras simultaneamente flexíveis e resistentes [4-6]. Além disso, eles são abundantemente cultivados em todo o mundo e oferecem a possibilidade de desenvolvimento social, uma vez que o cultivo pode ser uma fonte de renda para comunidades pobres em muitos países [7].

A fibra de Rami deve ser particularmente conhecida por ser uma das mais resistentes, o que pode atingir uma resistência à tração superior a $1000 \mathrm{MPa}$. Além disso, os compósitos de matriz epóxi reforçados com fibras rami atingiram 102,26 MPa para $30 \%$ de fibras em testes de tração [8-10]. Com o objetivo de melhorar ainda mais o desempenho dos compósitos de fibras naturais, é investigado o desempenho de compósitos reforçados com tecido rami (Figura 1), em vez de suas fibras alinhadas. O tecido reforça a matriz em mais de uma direção simultaneamente e também permite a introdução de uma maior fração de material de reforço. Esta nova estrutura, em relação ao uso do tecido rami, já foi testada para painéis e casacos à prova de balas [11].

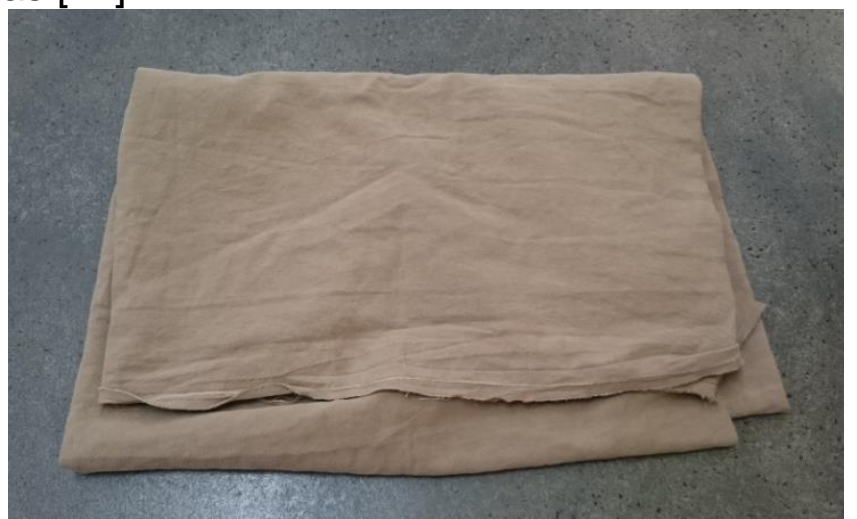

Figura 1 - Tecido Rami como recebido no laboratório

O objetivo deste trabalho é avaliar o comportamento de tração de matriz de matriz epóxi reforçada com tecido de rami.

\section{MATERIAIS E MÉTODOS}

O tecido usado para este trabalho foi obtido da empresa chinesa Rose Natural Healthy Items Wholesale. Os espécimes com diferentes frações de volume de tecido foram individualmente confeccionados incorporando camadas de tecido 1, 2 e 3 no compósito. Para isso, o tecido foi inicialmente cortado no formato do molde e depois pressionado com a resina líquida (Epóxi + DEGEBA / TETA) para garantir uma impregnação total. Depois disso, a quantidade suficiente de resina epóxi foi vertida 
no molde e depois as camadas de tecido foram colocadas sobre ela alternadamente com novas camadas de resina. Os espécimes foram curados durante 24 horas e testados em uma máquina universal Instron, modelo 5582, a $298 \mathrm{~K}\left(25^{\circ} \mathrm{C}\right)$. Os espécimes de tração são mostrados na Figura 2.

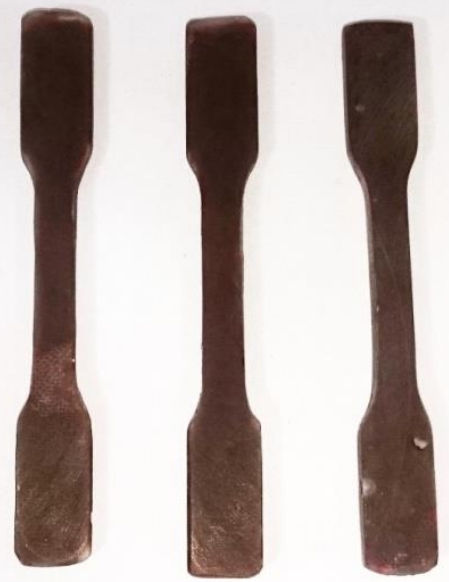

Figura 2 - espécimes de tração

\section{RESULTADOS E DISCUSSÃO}

As curvas típicas de carga e alongamento para o epóxi puro (tecido de $0 \%$ e compósitos diferentes são exemplificadas na Figura 3. Essas curvas foram registradas diretamente do software Instron. Após uma linha elástica reta, ocorre uma fratura súbita, indicando um comportamento frágil tanto para os espécimes de resistência à epóxi puro quanto para os compósitos de tecido de rami. 


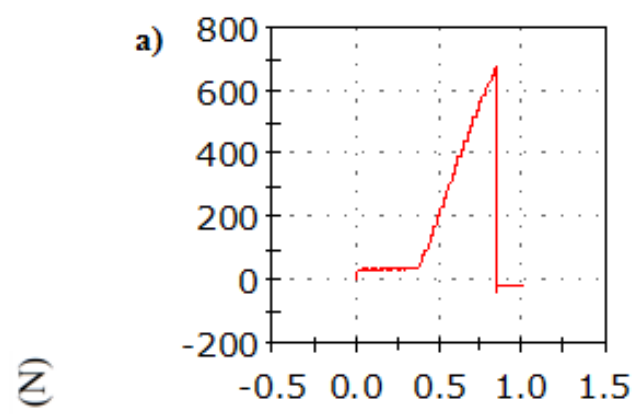

b)
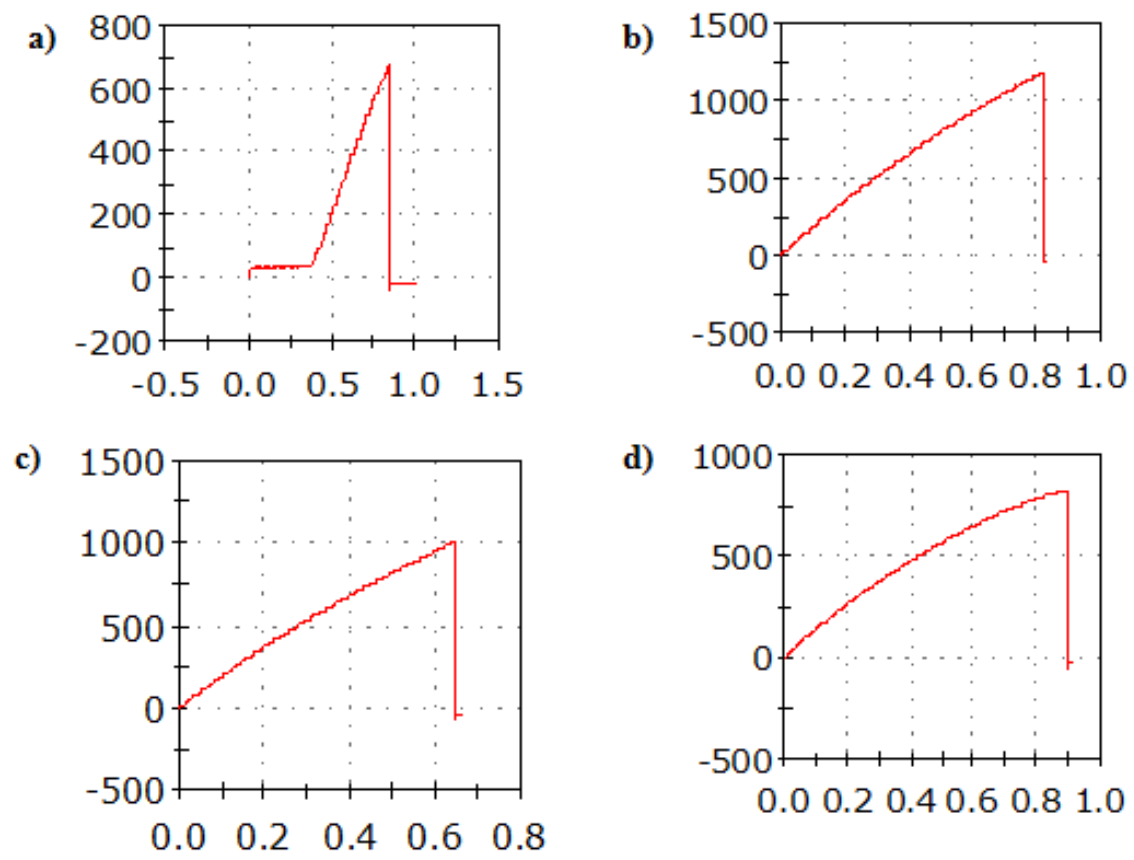

d)

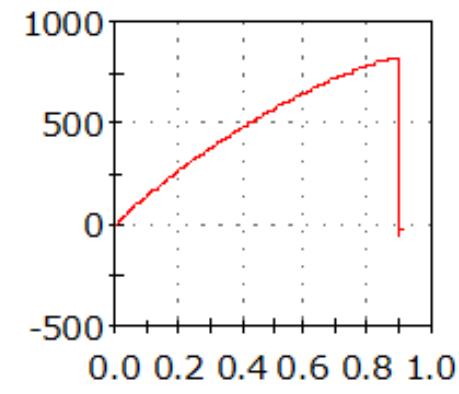

\section{Elongation $(\mathrm{mm})$}

Figura 3 - Curvas de carga vs. alongamento: (a) epóxi puro, (b) 1 camada de tecido, (c) 2 camadas de tecido e (d) 3 camadas de tecido.

A Figura 4 mostra o aspecto macro dos espécimes rompidos por tração correspondentes à diferente fração volumétrica do tecido rami. Nesta figura, a fratura de todos os espécimes tende a ser transversal ao eixo de tração. Não foram observadas evidências macroscópicas de participação do tecido rami, o que não revela efeito de retirada. Pode ser atribuído à configuração do tecido: enquanto as fibras paralelas ao eixo de tração são retiradas da matriz pela carga, as fibras perpendiculares atuam mantendo essas fibras na matriz. No entanto, mais estudos devem ser realizados para investigar mais profundamente esse efeito.

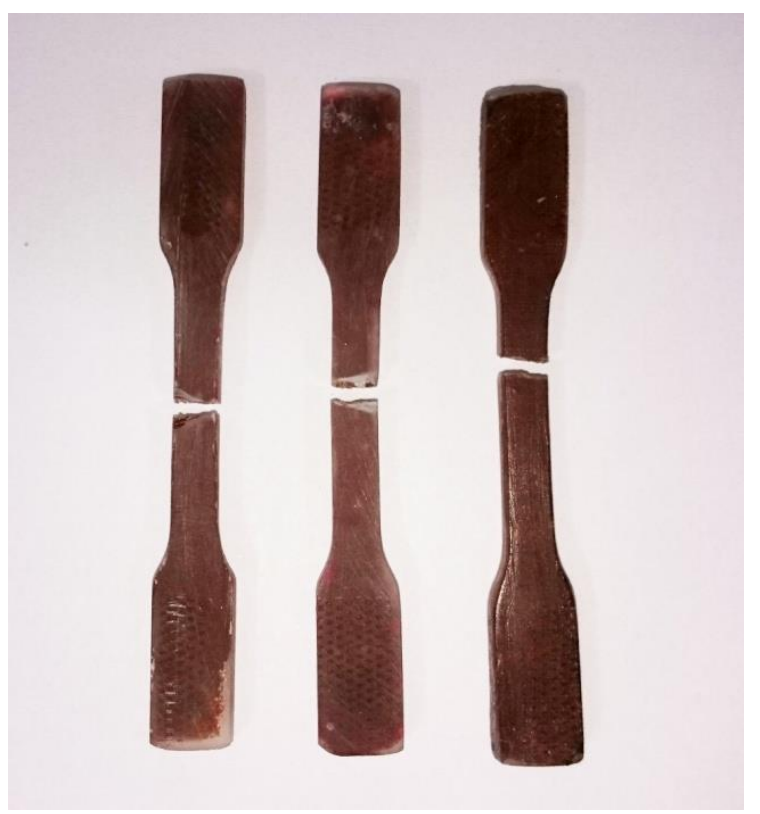

Figura 4 - espécimes de tração rompidos 
A partir dos dados observados nas curvas mostradas na Figura 3, foi possível obter a força de tração média (tensão máxima) e o módulo de elasticidade para diferentes frações volumétricas do tecido rami. Estes dados são apresentados na Tabela 1. Os resultados mostram que a presença de tecido rami aumenta a resistência à tração composta e quase não tem influência na rigidez.

Tabela 1 - Propriedades de tração de composições epóxi reforçadas com diferentes frações volumétricas de tecido rami

\begin{tabular}{|c|c|c|}
\hline $\begin{array}{c}\text { Número de camadas } \\
\text { de tecido }\end{array}$ & $\begin{array}{c}\text { Resistência à tração } \\
(\mathrm{MPa})\end{array}$ & $\begin{array}{c}\text { Módulo Elástico } \\
(\mathrm{GPa})\end{array}$ \\
\hline 0 & $24,15 \pm 3,88$ & $1,851 \pm 0,011$ \\
\hline 1 & $31,41 \pm 3,57$ & $1,844 \pm 0,011$ \\
\hline 2 & $34,24 \pm 3,09$ & $1,830 \pm 0,012$ \\
\hline 3 & $37,63 \pm 2,06$ & $1,827 \pm 0,011$ \\
\hline
\end{tabular}

A Figura 5 mostra os resultados gráficos para a variação da resistência à tração e do módulo de elasticidade com a fração volumétrica do tecido de rami. Pode ver-se que a resistência à tração (Figura 5a) aumenta razoavelmente, dentro das barras de erro, de maneira quase linear com o número de camadas de tecido. Representa um efeito de reforço considerável do tecido na matriz. De fato, o aumento da resistência à tração é de aproximadamente $55 \%$. Também é possível observar que o módulo de elasticidade (Figura 5b) quase não é afetado pela presença de tecido, diminuindo aproximadamente $2 \%$ no compósito com três camadas de tecido.
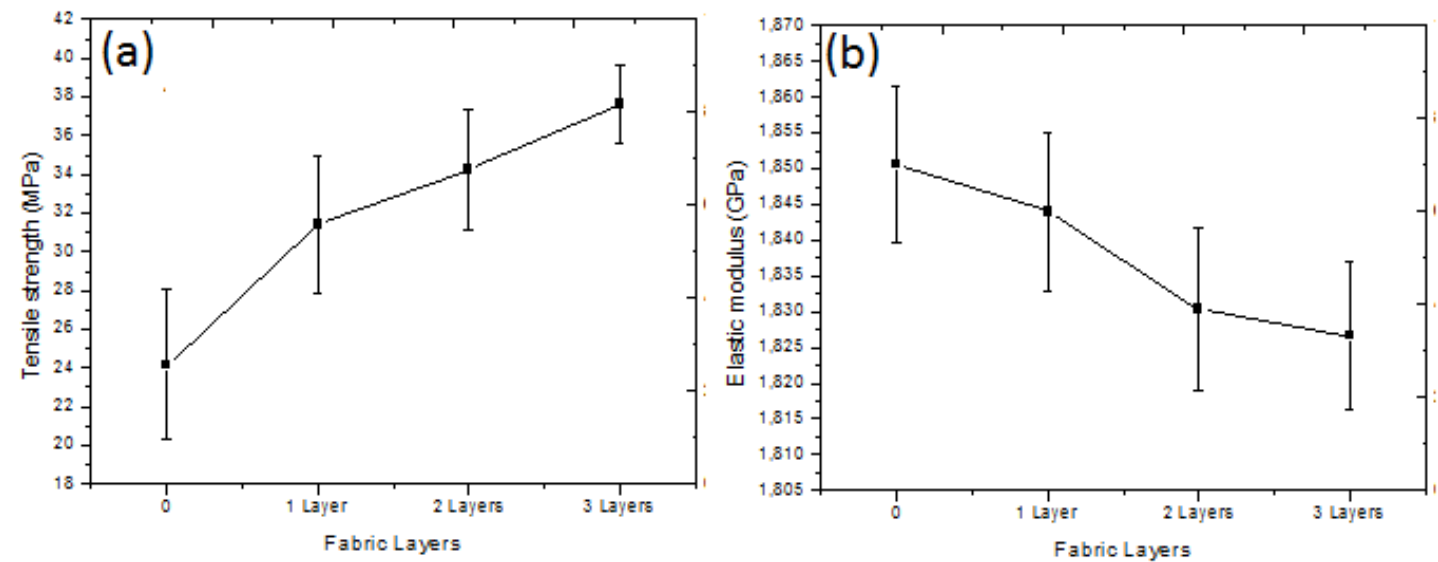

Figura 5 - Resultados gráficos de (a) resistência à tração (b) módulo de elasticidade para compósitos com diferente fração de volume de tecido de rami

Na Figura 6 é possível ver as fotografias em MEV da superfície da fratura. Observa-se que o tecido puxa ligeiramente para fora da matriz. Isto é devido ao efeito de delaminação, comum em compósitos reforçados com materiais laminados, como tecidos de fibras naturais. No entanto, o efeito de delaminação não é altamente intenso em compósitos submetidos a tensões de tração, portanto, mesmo com esse efeito, o tecido ainda mantém uma adesão razoável à matriz. Também pode ser observado, com base nas marcas de fratura na superfície da matriz epóxi, que o tecido funcionou como uma barreira à propagação de fissuras, aumentando a resistência à tração composta em comparação com o valor correspondente de epóxi puro. 

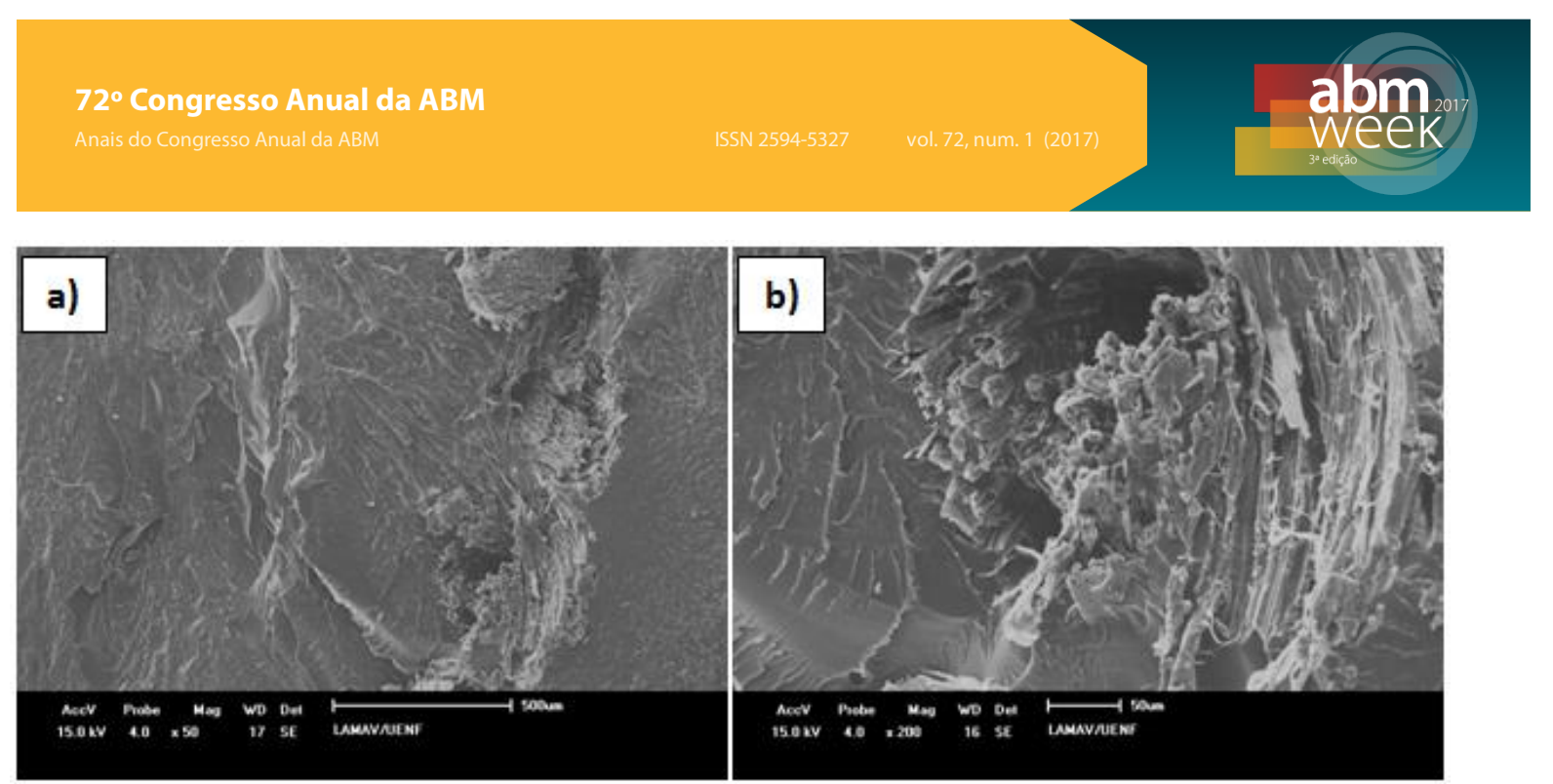

Figura 6 - Fotografias em MEV de uma amostra composta de epóxi com ruptura de tração com 3 camadas de tecido de rami: (a) $50 \mathrm{X}$ e (b) $200 \mathrm{X}$

\section{CONCLUSÃO}

- Os compósitos epóxi testados por tração reforçados com tecido rami exibem um aumento razoável na resistência à tração e quase nenhuma variação no módulo elástico.

- Com três camadas de tecido, a resistência à tração aumenta aproximadamente 55\%, enquanto 0 módulo elástico diminui aproximadamente $2 \%$ em comparação com valores correspondentes de epóxi puro.

- Embora o tecido forneça reforço multidirecional e permita a introdução de frações de maior volume de material de reforço, ele também é submetido a efeitos de delaminação e desengate, o que pode mitigar o efeito de reforço.

- A análise de fotografia em MEV revela que, apesar de um ligeiro efeito de delaminação, o tecido tem uma adesão razoável à matriz, trabalhando como barreira à propagação de fissuras e reforçando a matriz.

\section{Agradecimentos}

Os autores agradecem o apoio das agências brasileiras: CNPq, FAPERJ e CAPES para o desenvolvimento deste trabalho.

\section{REFERÊNCIAS}

1 A.K. Mohanty, M. Misra and L.T. Drzal: J. Polym. Environ., 2002, vol. 10, pp. 19-26.

2 A.N. Netravali and S. Chabba: Mater Today., 2003, vol. 6, pp. 22-29.

3 J. Crocker: Mater. Technol., 2008, vol. 2-3, pp. 174-8.

4 S.N. Monteiro, F.P.D. Lopes, A.S. Ferreira and D.C.O. Nascimento: JOM, 2009, vol. 61, pp. 17-22.

$5 \quad$ M.J .John and S. Thomas: Carbohydr. Polym., 2008, vol. 71, pp. 343-64.

6 S.N. Monteiro, F.P.D. Lopes, A.P. Barbosa, A.B. Bevitori, I.L.A. Silva and L.L.Costa: Met. Mat. Trans. A, 2011, vol. 42, pp. 2963-74.

7 O. Faruk, A.K. Bledzki, H.P. Fink and M. Sain: Progr. Polym. Sci. 2012, vol. 37, pp. 1555-96.

8 V.K. Thakur, M.K. Thakur and R.K. Gupta: Intl. J. Polym Anal. Charact., 2014, vol. 19, pp. 256-71.

9 A. Pappu, V. Patil, S. Jain, A. Mahindrakar, R. Haque and V.K. Thakur: Int. J. Biol. Macromol.: 2015, vol. 79, pp. 449-58. 
10 O. Güven, S.N. Monteiro, E.A.B. Moura and J.W. Drelich: Polym. Reviews., 2016, vol. 56, pp. 702-736.

11 T. L. Milanezi: Major Degree Dissertation, Military Institute of Engineering, 2015. 